\title{
Indoor Cardiovascular Health Monitoring System under COVID 19 Situations
}

\author{
Alok Mukherjee ${ }^{1}$, Deboleena Sadhukhan ${ }^{2}$, Kingshuk Chatterjee ${ }^{1}$, Tanmay Sarkar 3,* (D) \\ Government College of Engineering and Ceramic Technology, Kolkata \\ Applied Mechanics Department, IIT Madras, India \\ Malda Polytechnic, West Bengal State Council of Technical Education, Government of West Bengal, Malda, India \\ Correspondence: tanmays468@gmail.com (T.S.);
}

Scopus Author ID 57203373596

Received: 20.06.2021; Revised: 25.07.2021; Accepted: 29.07.2021; Published: 9.08.2021

\begin{abstract}
In this article, we propose a health monitoring system, especially focused on detecting myocardial infarction chances and raising the alarm when required. The device would be able to identify malfunctioning of the heart using the ECG signals taken using ARDUINO-based portable and affordable sensors. Here, we have described the development of two major schemes of health monitoring. We have developed a method of identifying the maloperation of the heart by using the second lead of the ECG signal. This method is based on a modified Poincaré analysis, which effectively distinguishes the diseased heart ECG from the normal heart ECG signals. The Poincaré-based scheme is tested using five different diseased ECG signals from the PTB database, and a high disease identification efficiency of $82 \%$ is achieved. Here we further propose implementing this analysis within the ARDUINO-based health monitoring board so that patients would get an opportunity to monitor their heart condition at some regular intervals staying at home and follow up with a physician if any abnormality is detected. This entire scheme is more useful, especially under this COVID 19, pandemic situation when moving outside the home is a challenge in itself. Hence, the proposed device would be helpful during this global crisis, especially for people residing in rural and semi-urban regions.
\end{abstract}

Keywords: home monitoring system; COVID 19; ECG; Poincaré plot; myocardial infarction (MI)

(C) 2021 by the authors. This article is an open-access article distributed under the terms and conditions of the Creative Commons Attribution (CC BY) license (https://creativecommons.org/licenses/by/4.0/).

\section{Introduction}

A COVID 19 global pandemic has given rise to several obstacles in the daily life of people. The pandemic is threatening people's health and taking away numerous lives every day $[1,2]$. Besides this lethal threat of this pandemic, many people are suffering from several other diseases, and many more people are even frightened to go out to health care centers for a medical checkup, even though they become doubtful regarding their health status [3,4]. Many of them are unaware that something wrong has happened to their health already as they are panic-stricken to go out in this crisis time. Our proposed health monitoring system is an ideal device for those people who want to study their health condition without moving outdoors. In such a situation, the proposed stand-alone low-cost health monitoring system will inform patients about their health parameters to detect and identify a person's basic heart status and inform health care personnel in case of any emergency. Many of the existing health care devices are not cost-effective [5]. Moreover, mostly they are not provided with any communication facilities to send urgent information to some preassigned person in case of any emergency [6- 
8]. Hence, the objective of this work is to propose an affordable home monitoring scheme that would be able to cater to a vast population of our country with a high level of accuracy and could be afforded by a mass of people from the safety of their home, especially during the present COVID 19 crisis $[9,10]$.

The objective of this work is twofold. In the first phase of this work, we have developed a scheme of identifying the human heart's maloperation and find a rough measure of the extent of illness caused to it based on only the 2nd lead data of the ECG signals. Scientists have worked over the years to achieve high accuracy in classifying myocardial infarction (MI) diseases $[11,12]$. Automated myocardial infarction identification is in existence for a long time. But most of the methods, although accurate, employ computationally intensive methods [13]. The proposed work is intended to develop an easy and effective method that would be highly effective to implement in the home monitoring device. The primary intention is to develop an algorithm for identifying myocardial infarction diseases, which would be simple enough to occupy less memory space and require less volume of ECG signal for transmission with low data band. Hence, concentration has been paid to use a single lead (lead II) ECG signal only for analysis using this method, sacrificing detection accuracy to some extent, compared to contemporary existing detection topologies [14]. Most of the existing researches use tools such as variants of supervised learning algorithms like a neural network, or rigorous mathematical approaches like Wavelet transform or WT, fuzzy inference system or FIS, or the latest development like machine learning or deep neural network, which are computationally much heavier compared to the proposed method. Ansari et al. [15] and Sharma et al. [16] provide a detailed review of several different techniques used in research. These methods are generally composed of four major steps: preprocessing, ECG beat segmentation, feature extraction, and classification. However, the feature extraction and classification components play a vital role in determining their performance and computational overhead.

Only the 2nd lead data requirement makes the acquiring ECG device simpler and hence becomes cost-effective [17]. Standard PTB database has been used in this work as the ECG signals for analysis $[18,19]$. We have used 72 numbers of healthy ECG signals of 52 healthy subjects and developed a disease identifier algorithm based on a modified Poincaré analysis. Finally, we have validated the scheme using a diseased ECG signal of 50 patients randomly selected suffering from different myocardial infarction heart diseases. Simple image processing methodology is applied to observe the repetitiveness of the signal and the extent of overlap of each cycle of the signal. Thus the level of abnormality of the signal is obtained, which indicates the extent of disturbance from the healthy condition. The proposed model identifies diseases with an accuracy of $82 \%$.

Finally, we propose an ARDUINO-based basic health monitoring system that would help people stay at home yet continuously monitor their heart condition under this pandemic situation. The major idea is to implement the ECG heart abnormality detection method as discussed earlier in this ARDUINO-based health monitoring board to achieve direct detection of abnormality. The proposed stand-alone system, in this way, would be able to detect the basic heart condition of a person; simultaneously, this would notify the relatives of the person or the health care personnel in case of emergency using the inbuilt GSM module. Apart from ECG, some other basic physiological parameters like body temperature and heart rate would also be detected. The proposed model is more cost-effective than most of the similar devices available. Most importantly, one such compact device has rarely been developed at an affordable cost. 


\subsection{Different methodologies used for detection of myocardial infarction.}

Researchers have adapted to different techniques to detect and analyze the different heart diseases, among which myocardial infarction bears records of being one of the fatal diseases of the human heart. In this proposed work, we have directly concentrated on an effective method of identifying this heart disease, which would be simple enough for easy implementation in a home monitoring device, especially under this pandemic situation. Most of the MI diseases are detected using complex algorithms to achieve better accuracy; although in this article, we have paid more emphasis on the easy detection of the fault in the heart, using only a single lead ECG signal to lessen data length: hence enable fast transmission of the signal to the remotely located health centers. Extreme emphasis has been imposed on the methods involving supervised learning approaches like the artificial neural network or ANN. The latest advancement of soft computational techniques has helped evolve numerous neural computational strategies, among which machine learning and deep learning methods bear significant importance. Hybrid forms of neural networks with different classifying techniques like FIS have been imposed tremendous importance [20]. The combination of mathematical approaches like WT and learning algorithms like support vector machines or SVM has been largely influential in developing disease detection algorithms, especially for the identification of MI disorders [21], which is also investigated here. Other methods like tensor-based analysis [22] and several other deep learning-based methodologies have been practiced widely. These methods are extremely accurate in detecting heart diseases of different classes, although each of these suffers from the respective drawbacks. Supervised learning methods like ANN are mostly dependent on accurate training methods $[23,24]$ involving large and diverse training signals; hence have high associated training time. WT-based methods become progressively intricate, especially with a high level of decomposition of the signal. Besides, the selection of the application-specific mother wavelet also possesses minor issues in occasional cases. Development of the classifier rule bases for FIS methods often opens up spaces for inaccuracies. Despite these hindrances, the above methods, especially the supervised approaches involving neural network has been paid major attention.

Preprocessing the signals is another issue in most of the works, which introduces more complexity to the analysis. Preprocessing of most of the techniques, especially in earlier research, mostly relies on feature extraction using the complete or partial QRS complex of the signal [25-28]. Researchers have used multiple analysis methods to serve the purpose of MI classification [27]. They have employed four major pattern-recognition approaches like Radial Basis Function, Probabilistic Neural Network or PNN, k-Nearest Neighbors (KNN), and Naive Bayes Classification. Few researchers have also emphasized feature extraction using Discrete Wavelet Transform (DWT) and Principal Component Analysis (PCA), followed by analyzing the so extracted features using variants of neural networks [29,30]. PCA is another useful tool for reducing a large dimension of data set into the most important directions of variation of the signal, which, in turn, reduces the data set, enabling lesser occupancy of memory and higher speed data transmission. However, the assumption of linearity of the principal components introduces some level of inaccuracies in the system. Wavelet analysis extracts the frequency components even from the fractional wavelengths, although it is often disturbed by high noise in the signals. Scholars have further used two variants of neural networks: Shallow convolutional neural network and end-to-end deep residual learning with dilated convolutions [31]. 
Some of the other works have employed ECG beats [32] of the entire 12 lead ECG signals itself [33] to use with Convolution Neural Network or CNN [32], which have become an extremely important variant of the neural network family, as well as using a combined CNN and RNN based structure. Another effective combination of CNN and RNN combined architecture has been proposed in [34], which have used Heart rate and beat interval-based features to detect and classify heart rate abnormalities. An RNN based model has been proposed in [35] using down-sampled ECG signals, whereas a fair comparison of the three different supervised learning models: Convolutional, Residual, and Inception neural network has been illustrated in [36]. Application image processing, applied on the grayscale image formed using the ECG signals, followed by analysis with transfer learning techniques, is illustrated in [37]. Another transfer learning-based approach is proposed in [38], which uses two-channel ECG signals for analysis.

Deep learning has been a tremendous influence in this field of study as in all other research disciplines. A deep learning-based approach has been proposed in [39], which uses a two-second long signal window and processes the data using a 16-layer CNN-LSTM deep learning model. A similar approach to detecting abnormality is proposed in [40], which again uses a segmented window of four seconds, followed by an analysis of the same using a similar LSTM model. Deep learning approaches have been adopted by the researchers of [41], where a complex method of preprocessing has been carried out using Fourier-Bessel expansion based on WT. Using deep learning methods, a 12-lead ECG signal detection and location method is also proposed in [42].

As is observed from the above analysis that most of these methods employ intricate computational schemes using diverse topologies, mostly involving variants of neural network and deep learning, often associated with other features extraction tools like Wavelet Transform, Principal Component Analysis, Fourier based analysis, and several others. In contrast, the proposed method doesn't use any major preprocessing of the signal, and the detection algorithm involves only difference signal-based Poincaré plot analysis to obtain a measure of pixel density in the plot. This method is extremely simple in analysis; hence possesses the necessary absence of major computational burden, which is extremely important for implementation in a home monitoring device.

\section{Materials and Methods}

\subsection{Methodology of ECG signal analysis.}

\subsubsection{Source of data set used in this work.}

In this research work, PTB database has been used to obtain the ECG signals of the healthy and the diseased patients [18,19]. Physikalisch-Technische Bundesanstalt (PTB), the National Metrology Institute of Germany, has provided a large compilation of digitized ECG signals for various purposes such as research, algorithmic benchmarking, or teaching for the users of PhysioNet. The signals have been collected from a number of healthy volunteers and patients having various heart diseases. The proposed work has been intended to detect different myocardial infarction (MI) disorders. The database contains 148 subjects of MI disease and 52 subjects with healthy controls. This work has investigated the signals of all the 52 normal subjects and a random sample of 50 subjects with MI disorders. Further, a total of 72 normal 
ECG signals containing multiple signals of a few subjects among the total number of 52 subjects with healthy controls have been studied as the normal or healthy set.

\subsection{Steps of development of the algorithm.}

We have used only the lead 2 data of the complete ECG signal for the analysis in the proposed analysis. This lead 2 signal $\mathrm{f}(\mathrm{n})$ is analyzed using modified Poincaré analysis.

Step1: $f(n)$ is first filtered using a low pass filter to eliminate most of the very highfrequency noise components. The filter's cutoff frequency is kept such that it is directed to majorly remove the noise component of the signal and retain most of the vital information without modifying the ECG signal.

Step 2: This signal is next median filtered to perform the baseline corrections, thus producing the unified signal.

Step 3: Producing difference signal fdiff (n)by making the following analysis: fdiff (n) $=\mathrm{f}(\mathrm{n})-\mathrm{f}(\mathrm{n}-1)$.

Step 4: Plotting the difference signal against the original signal, i.e., plotting fdiff (n) against each point $\mathrm{f}(\mathrm{n})$. Thus a modified Poincaré plot is obtained.

Step 5: This plot is obtained as an image which is analyzed further.

The idea is to obtain the measure of the repetitiveness of the signal. The ECG signal is captured for a complete 1 minute, and each of the periods of the signal is studied. It is found that if the heart is working under normal conditions, each of the periods of the ECG signal is almost identical. Hence, when the two variables fdiff (n) and $f(n)$ are plotted against each other, the near-identical repetitiveness of the individual periods of the normal heart signal develops the plot with very narrow thickness, as most of the periods of the signals overlap on each other. Eight such images with normal heart signals are shown in Figure 1. It is well observed that the normal signals produce an almost overlapping image of the modified Poincaré plot. The diseased plots are also shown in Figures 2-6.

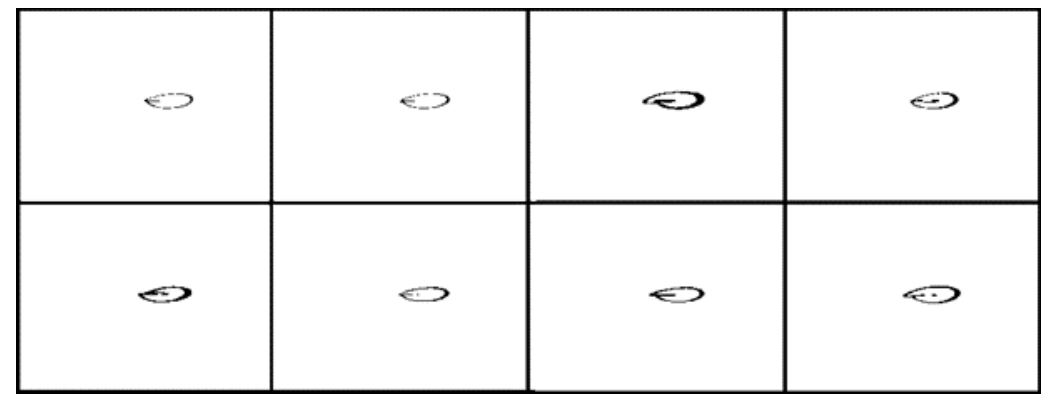

Figure 1. Modified Poincaré plot of eight random ECG signals of normal patients with no disease.

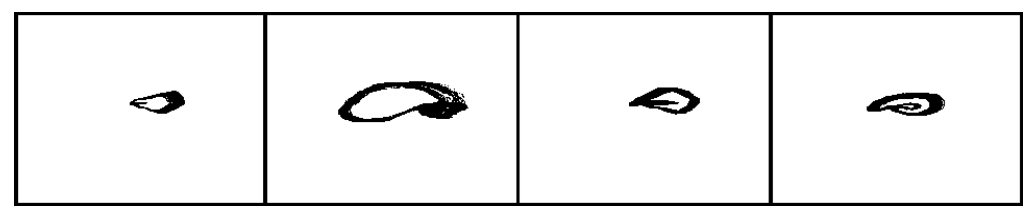

Figure 2. Modified Poincaré plot of four random ECG signals of normal patients with MI Anterior disease.

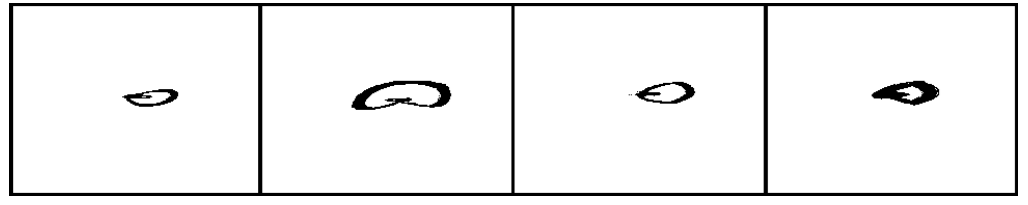


Figure 3. Modified Poincaré plot of four random ECG signals of normal patients with MI Anterior Lateral disease.

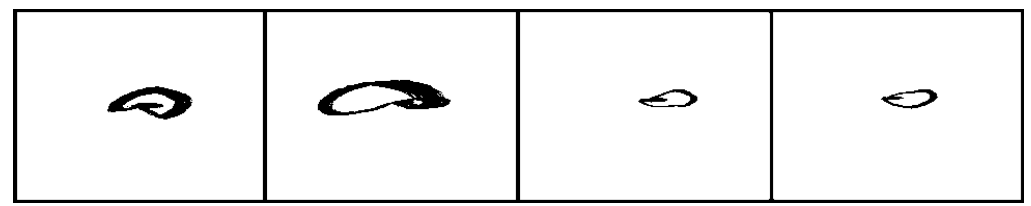

Figure 4. Modified Poincaré plot of four random ECG signals of normal patients with MI Anterior Septal disease.

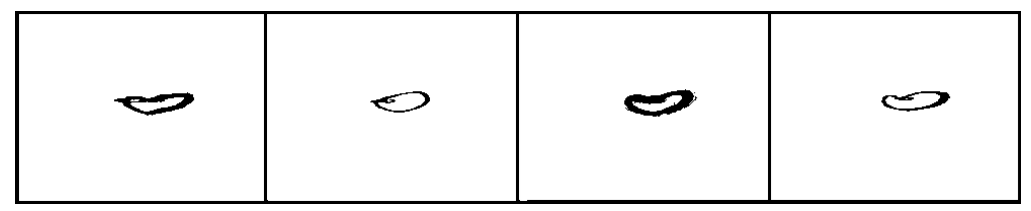

Figure 5. Modified Poincaré plot of four random ECG signals of normal patients with MI Inferior disease.

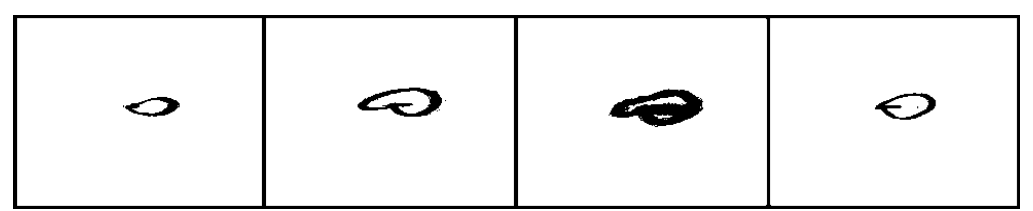

Figure 6. Modified Poincaré plot of four random ECG signals of normal patients with MI Inferior Lateral disease.

\subsection{Numerical analysis of Poincaré plots.}

Both the normal and the diseased signals are captured with the same frame of axes. Hence, comparing the same of the normal and the five different diseased plots, it is well observed that the diseased heart plots have much higher thickness compared to the normal plots. Hence, the number of pixels occupied by the diseased plots is much higher in number compared to the normal plots. Next, a simple image processing algorithm is applied over these modified Poincare plots to obtain the ratio of the number of black pixels to the number of white pixels. As mentioned earlier, normal ECG signals of 72 people along with diseased heart ECG signals of 50 patients are analyzed using the proposed method. On analyzing the images, the percentage ratio of the number of black pixels (B) to the number of white pixels (W) is computed with normal ECG signals, and the diseased ECG signals and are shown in Table 1 and Table 2, respectively. The percentage ratio $(\mathrm{R})$ is termed as overlap index subsequently in this article since this ratio indicates the extent of overlap of each cycle of the ECG signal. This index is given by the following expression:

Overlap Index $(\mu)=[$ Number of black pixels (B) / Number of white pixels $(\mathrm{W})] \times 100 \%$

Table 1. Computation of the ratio of black pixels to white pixels of modified Poincaré images of 72 normal patients.

\begin{tabular}{c|c|c|c|c|c|c|c|c|c|c|c}
$\mathbf{B}$ & $\mathbf{W}$ & $\boldsymbol{\mu}$ & $\mathbf{B}$ & $\mathbf{W}$ & $\boldsymbol{\mu}$ & $\mathbf{B}$ & $\mathbf{W}$ & $\boldsymbol{\mu}$ & $\mathbf{B}$ & $\mathbf{W}$ & $\boldsymbol{\mu}$ \\
\hline 1032 & 234168 & 0.44 & 801 & 234399 & 0.34 & 926 & 234274 & 0.4 & 1501 & 233699 & 0.64 \\
\hline 1059 & 234141 & 0.45 & 1161 & 234039 & 0.5 & 3032 & 232168 & 1.31 & 1641 & 233559 & 0.7 \\
\hline 2059 & 233141 & 0.88 & 1124 & 234076 & 0.48 & 1747 & 233453 & 0.75 & 1115 & 234085 & 0.48 \\
\hline 722 & 234478 & 0.31 & 1048 & 234152 & 0.45 & 1469 & 233731 & 0.63 & 874 & 234326 & 0.37 \\
\hline 718 & 234482 & 0.31 & 2464 & 232736 & 1.06 & 1626 & 233574 & 0.7 & 955 & 234245 & 0.41 \\
\hline 1316 & 233884 & 0.56 & 2519 & 232681 & 1.08 & 1648 & 233552 & 0.71 & 1471 & 233729 & 0.63 \\
\hline 1482 & 233718 & 0.63 & 2168 & 233032 & 0.93 & 3413 & 231787 & 1.47 & 1053 & 234147 & 0.45 \\
\hline 1991 & 233209 & 0.85 & 1906 & 233294 & 0.82 & 889 & 234311 & 0.38 & 881 & 234319 & 0.38 \\
\hline 1025 & 234175 & 0.44 & 2931 & 232269 & 1.26 & 782 & 234418 & 0.33 & 1804 & 233396 & 0.77 \\
\hline 1064 & 234136 & 0.45 & 2948 & 232252 & 1.27 & 1422 & 233778 & 0.61 & 4383 & 230817 & 1.9
\end{tabular}




\begin{tabular}{c|c|c|c|c|c|c|c|c|c|c|c}
$\mathbf{B}$ & $\mathbf{W}$ & $\boldsymbol{\mu}$ & $\mathbf{B}$ & $\mathbf{W}$ & $\boldsymbol{\mu}$ & $\mathbf{B}$ & $\mathbf{W}$ & $\boldsymbol{\mu}$ & $\mathbf{B}$ & $\mathbf{W}$ & $\boldsymbol{\mu}$ \\
\hline 860 & 234340 & 0.37 & 1805 & 233395 & 0.77 & 1313 & 233887 & 0.56 & 692 & 234508 & 0.3 \\
\hline 818 & 234382 & 0.35 & 1555 & 233645 & 0.67 & 1024 & 234176 & 0.44 & 1539 & 233661 & 0.66 \\
\hline 759 & 234441 & 0.32 & 2418 & 232782 & 1.04 & 1287 & 233913 & 0.55 & 1291 & 233909 & 0.55 \\
\hline 1147 & 234053 & 0.49 & 1113 & 234087 & 0.48 & 1564 & 233636 & 0.67 & 1405 & 233795 & 0.6 \\
\hline 1196 & 234004 & 0.51 & 980 & 234220 & 0.42 & 1091 & 234109 & 0.47 & 1271 & 233929 & 0.54 \\
\hline 1013 & 234187 & 0.43 & 1163 & 234037 & 0.5 & 736 & 234464 & 0.31 & 797 & 234403 & 0.34 \\
\hline 3125 & 232075 & 1.35 & 1179 & 234021 & 0.5 & 1171 & 234029 & 0.5 & 956 & 234244 & 0.41 \\
\hline 1376 & 233824 & 0.59 & 1254 & 233946 & 0.54 & 1946 & 233254 & 0.83 & 930 & 234270 & 0.4
\end{tabular}

Table 2. Computation of the ratio of black pixels to white pixels of modified Poincaré images of 50 diseased patients: 10 patients belong to each of the 5 diseases: MI Anterior, MI Anterior Lateral, MI Anterior Septal, MI Inferior and MI Inferior Lateral.

\begin{tabular}{c|c|c|c|c|c|c|c|c|c|c|c|c|c|c}
\multicolumn{4}{c|}{ MI_Anterior } & \multicolumn{3}{c}{ MI_Anterior_Lateral } & \multicolumn{3}{c|}{ MI_Anterior_Septal } & \multicolumn{3}{c|}{ MI_Inferior } & \multicolumn{2}{c}{ MI_Inferior_Lateral } \\
\hline $\boldsymbol{B}$ & $\boldsymbol{W}$ & $\boldsymbol{\mu}$ & $\boldsymbol{B}$ & $\boldsymbol{W}$ & $\boldsymbol{\mu}$ & $\boldsymbol{B}$ & $\boldsymbol{W}$ & $\boldsymbol{\mu}$ & $\boldsymbol{B}$ & $\boldsymbol{W}$ & $\boldsymbol{\mu}$ & $\boldsymbol{B}$ & $\boldsymbol{W}$ & $\boldsymbol{\mu}$ \\
\hline 2694 & 232506 & 1.16 & 3577 & 231623 & 1.54 & 6268 & 228932 & 2.74 & 3649 & 231551 & 1.58 & 2140 & 233060 & 0.92 \\
\hline 10410 & 224790 & 4.63 & 1935 & 233265 & 0.83 & 3032 & 232168 & 1.31 & 2194 & 233006 & 0.94 & 1488 & 233712 & 0.64 \\
\hline 4359 & 230841 & 1.89 & 1929 & 233271 & 0.83 & 9213 & 225987 & 4.08 & 4754 & 230446 & 2.06 & 4813 & 230387 & 2.09 \\
\hline 2595 & 232605 & 1.12 & 5252 & 229948 & 2.28 & 8523 & 226677 & 3.76 & 2099 & 233101 & 0.9 & 16924 & 218276 & 7.75 \\
\hline 9548 & 225652 & 4.23 & 1027 & 234173 & 0.44 & 9468 & 225732 & 4.19 & 3036 & 232164 & 1.31 & 12203 & 222997 & 5.47 \\
\hline 1977 & 233223 & 0.85 & 2998 & 232202 & 1.29 & 2944 & 232256 & 1.27 & 1751 & 233449 & 0.75 & 7251 & 227949 & 3.18 \\
\hline 4916 & 230284 & 2.13 & 1583 & 233617 & 0.68 & 1854 & 233346 & 0.79 & 1631 & 233569 & 0.7 & 9778 & 225422 & 4.34 \\
\hline 1514 & 233686 & 0.65 & 2441 & 232759 & 1.05 & 1640 & 233560 & 0.7 & 1480 & 233720 & 0.63 & 1841 & 233359 & 0.79 \\
\hline 1542 & 233658 & 0.66 & 4253 & 230947 & 1.84 & 1785 & 233415 & 0.76 & 1359 & 233841 & 0.58 & 11384 & 223816 & 5.09 \\
\hline 9010 & 226190 & 3.98 & 1815 & 233385 & 0.78 & 1809 & 233391 & 0.78 & 9925 & 225275 & 4.41 & 2646 & 232554 & 1.14
\end{tabular}

These values were further analyzed using box plots, which are shown in Figure 7. The quartile ranges and median values are also described in Table 3. These box plots are the plot of the percentage ratio $(\mathrm{R})$ with respect to the different diseases and the normal plots. It is well observed that apart from some of the outliers, the median value of the percentage ratio of the normal plots is lying much below the median value of all other diseased plots, which creates the major source of differentiation of the normal and the diseased patients. Moreover, it is further encouraging to observe that the upper boundary of the normal box plot's upper quartile lies much below even the lower boundary of the lower quartile of all the diseased cases. This fact dominantly emphasizes the distinction obtained among the modified plots of the diseased and non-diseased patients. This, in turn, establishes the superiority of this simple scheme for ECG analysis for disease detection.

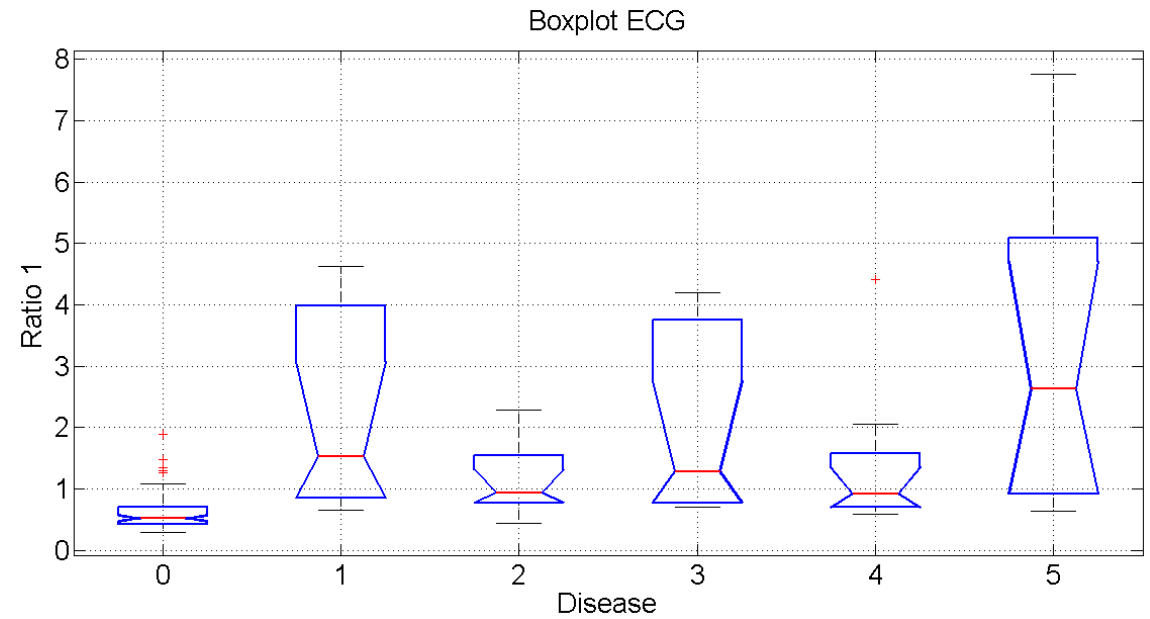

Figure 7. Percentage ratio (R) values of normal and diseased cases obtained from modified Poincaré plots; Disease sequence are as follows: 0: Normal, 1: MI Anterior, 2: MI Anterior Lateral, 3: MI Anterior Septal, 4: MI Inferior and 5: MI Inferior Lateral. 
Table 3. Ranges of quartile deviation of normal and five different diseases.

\begin{tabular}{c|c|c|c|c} 
Disease Name & $\begin{array}{c}\text { Disease } \\
\text { Index }\end{array}$ & $\begin{array}{c}\text { Lower quartile } \\
\text { limit }\end{array}$ & Median & $\begin{array}{c}\text { Upper quartile } \\
\text { limit }\end{array}$ \\
\hline Normal & 0 & 0.4255 & 0.5235 & 0.7041 \\
\hline MI Anterior & 1 & 0.8477 & 1.5235 & 3.9834 \\
\hline MI Anterior Lateral & 2 & 0.7777 & 0.9391 & 1.5443 \\
\hline MI Anterior Septal & 3 & 0.7751 & 1.2868 & 3.76 \\
\hline MI Inferior & 4 & 0.6983 & 0.921 & 1.5759 \\
\hline MI Inferior Lateral & 5 & 0.9182 & 2.6351 & 5.0863
\end{tabular}

\section{Results and Discussion}

The proposed disease detection scheme is developed using a simple comparative analysis of the percentage ratio $(\mathrm{R})$, values obtained from analyzing the modified Poincaré images. This simple technique makes it very efficient to implement in a home monitoring system. The upper limit of the upper quartile is chosen as the limiting threshold value $(\phi)$, which is found as 0.7041 . Hence, it is ascertained that $75 \%$ of the total normal patients have this index less than $\phi$. The R-value of the unknown patient is compared with this threshold to obtain a simple classifier algorithm. If the R-value of the unknown patient is above this threshold, the patient is denoted as diseased or normal. This method of classification produced the following results of disease detection, which are shown in Table 4.

It is further required to mention here that the patients would be able to identify the extent of disorder of their heart by comparing the overlap index obtained from the ECG signal of their heart and that of the maximum reference value; by looking at the inbuilt display of the proposed device where both these values would be displayed. The above analysis shows that the upper range of the overlap index of its $25 \%$ to $75 \%$ quartile range is found as 0.4255 to 0.7041 . Hence, the patient would be able to develop an idea of their heart condition by comparing their overlap index with this reference range. This would work as an initial screening for the patient. If the same index exceeds the upper quartile highest level of 0.7041 , the device immediately sends SMS to the concerned person.

Table 4. Accuracy of the proposed algorithm identifying different diseases.

\begin{tabular}{c|c|c|c|c} 
Disease Name & $\begin{array}{c}\text { Number } \\
\text { of cases }\end{array}$ & $\begin{array}{c}\text { Number of correct } \\
\text { detection }\end{array}$ & $\begin{array}{c}\text { Number of the } \\
\text { wrong detection }\end{array}$ & $\begin{array}{c}\text { Percentage of } \\
\text { correct detection }\end{array}$ \\
\hline MI Anterior & 10 & 8 & 2 & 80 \\
\hline MI Anterior Lateral & 10 & 8 & 2 & 80 \\
\hline MI Anterior Septal & 10 & 9 & 1 & 90 \\
\hline MI Inferior & 10 & 7 & 3 & 70 \\
\hline MI Inferior Lateral & 10 & 9 & 1 & 90 \\
\hline Overall Accuracy & $\mathbf{5 0}$ & $\mathbf{4 1}$ & $\mathbf{9}$ & $\mathbf{8 2}$
\end{tabular}

\subsection{Design of the proposed health monitor scheme.}

The proposed ECG analysis scheme is intended to be implemented in an ARDUINObased automatic health monitoring system, especially for this pandemic situation when staying indoors is of utmost importance. The proposed health monitoring system is designed to detect the basic health condition regarding ECG, temperature monitor, and heart rate. Another key feature of this design would be its ability to notify the relative or the health care personnel in an emergency using the inbuilt basic GSM module. The proposed design would be compact in measuring these important parameters using a single device. Most importantly, the costeffectiveness of the device would count for its effectiveness, especially under this crisis period. 


\subsection{Design specifications.}

The system is designed on ARDUINO MEGA 2560 board with the physiological signal acquisition modules interfaced. Another ARDUINO UNO R3 board is connected for developing the communication device, which is interfaced with a GSM module. A basic processing program is developed and run in ARDUINO IDE. This is further given to the onboard memory for processing the acquired signals and generate an alarm if necessary. A display module is also connected to the system, displaying the basic measured parameters and values.

i) The inbuilt GSM module is provided with a SIM. This is used for notifying the relatives or the medical persons directly through SMS about any emergency.

ii) A LED and a buzzer are also connected to generate visual attention and sound alarm in an emergency.

iii) The temperature sensor connected to the module measures the body temperature of the patient. The sensed temperature signal is converted to Fahrenheit in the program and displays on the screen. It similarly communicates with the GSM module and generates an alarm if the temperature goes beyond $101 \mathrm{~F}$. The program also intimates the patients if he/she does not record temperature for more than 8 hours at a stretch and generates an alarm for the patient itself. If still unattended, the module sends an SMS.

iv) The ECG signal acquisition and analysis module is the most important part of the proposed health monitoring device. The AD8232 based ECG (Electro-cardiogram) acquisition module is used in this work to acquire single lead ECG data of the patient $[43,44]$. This method uses three leads connected to the patient's left and right arm and right leg. The embedded program analyzes the acquired ECG as per the proposed algorithm discussed formerly. The proposed analysis detects the cardiac abnormalities as per the previously described overlap index-based heart malfunction detection scheme. The maximum level of reference overlap index and that of the patient is displayed in the inbuilt display module, which indicates the extent of deviation of the patient's heart from the photoplethysmography normal value [45]. In case if the overlap index exceeds the prescribed maximum reference level, the GSM module again communicates with the concerned persons.

v) The Easy pulse sensor module is also inbuilt in the device using HRM-2511E transmission PPG (Photoplethysmogram) sensor [46]. This is used for monitoring the heart rate of the patient by counting the number of PPG peaks. The GSM module is also flagged in case of any abnormality.

vi) Another pressure sensor is also placed underneath the patient's bed. This sensor is used to count the duration for which the patient is lying on the bed. In case if the duration is alarmingly low or high, the display and alarm indicate the patient is concerned, and if untreated for the next one hour, the GSM module is triggered to send SMS to the concerned medical person.

\subsection{Usefulness of the proposed design.}

The design of the proposed health monitoring device is portable and built on ARDUINO-based platform. The design is made using ARDUINO microcontrollers with some targeted sensors which are embedded in a compact system. The proposed device is easy to use, simple, portable and cheap. Most importantly, the proposed device can reach out to even the 
rural population where the benefits of health care, as well as internet service, are not state-ofthe-art. This device will be extremely helpful for rural and urban populations, especially under this pandemic situation, where even reaching out to healthcare centers is a challenge.

The device is easy to install and is able to operate without the intervention of an expert or health personnel. Such a device will definitely aid the day-to-day health monitoring of the closed-door people in this global pandemic crisis. This device will especially be helpful for the elderly population. Another important feature of this device is offered by the inbuilt GSM module, which is designed to communicate with the preassigned relatives or the medical personnel by timely informing through SMS on detection of any abnormality in any of the above-mentioned measuring parameters. This can largely reduce the hazards of being late in informing physicians. The model itself is less costly compared to some of the contemporary devices. The cost would further come down on a large-scale production post popularization of the device. A huge number of the people from both rural and urban parts of the country would be benefitted from this device, especially under the pandemic stricken people of the country. Further, this would also allow the close kins of especially the patients or the older people, to be assured of the security of their close ones.

\section{Conclusions}

This article has developed a computationally simple technique to identify myocardial infarctions (MI) using lead 2 data of an ECG signal, analyzed using a modified Poincaré plot and simple image processing technique. The proposed method doesn't include any major preprocessing of the signal. The detection algorithm involves only difference signal-based Poincaré plot analysis to measure pixel density in the plot. This method is extremely simple in analysis; hence possesses the necessary absence of major computational burden, which is extremely important for implementation in a home monitoring device. Apart from the MI detection algorithm, the proposed home monitoring device is able to raise the alarm when a diseased ECG is identified. The simplicity of the technique makes it an ideal candidate for implementation in a health monitoring system, which is extremely important under the current scenario of the COVID 19 crisis, where direct medical intervention is difficult to achieve. We have also proposed a cost-effective health monitoring system that will employ the above technique. Altogether, the proposed MI detection technique and possible implementation in the proposed home monitoring device suggest the possibility of developing a low-cost and suitable home-healthcare device.

\section{Funding}

Not applicable.

\section{Acknowledgments}

Not applicable.

\section{Conflicts of Interest}

The authors declare no conflict of interest. 


\section{References}

1. Monajjemi, M.; Shahriari, S.; Mollaamin, F. Evaluation of coronavirus families \& COVID-19 proteins: Molecular modeling study. Biointerface Res. Appl. Chem. 2020, 10, 6039-6057, https://doi.org/10.33263/BRIAC105.60396057.

2. Dhumad, A.M.; Harismah, K.; Zandi, H. Interacting cubane assisted bi-cytidine with covid-19 main protease: In silico study. Biointerface Res. Appl. Chem. 2021, 11, 13962-13967, https://doi.org/10.33263/BRIAC116.1396213967.

3. Hasan, M.R.; Paul, B.K.; Ahmed, K.; Mahmud, S.; Dutta, M.; Hosen, M.S.; Hassan, M.M.; Bhuyian, T. Computational analysis of network model based relationship of mental disorder with depression. Biointerface Res. Appl. Chem. 2020, 10, 6293-6305, https://doi.org/10.33263/BRIAC105.62936305.

4. Al-Masoudi, N.A.; Elias, R.S.; Saeed, B. Molecular docking studies of some antiviral and antimalarial drugs via bindings to 3cl-protease and polymerase enzymes of the novel coronavirus (Sars-cov-2). Biointerface Res. Appl. Chem. 2020, 10, 6444-6459, https://doi.org/10.33263/BRIAC105.64446459.

5. Rahaman, A.; Islam, M.; Islam, R.; Sadi, M..; Nooruddin, S. Developing iot based smart health monitoring systems: A review. Rev. d'Intelligence Artif. 2019, 33, 435-440, https://doi.org/10.18280/ria.330605.

6. Kumar, S.P.; Samson, V.R.R.; Sai, U.B.; Rao, P.L.S.D.M.; Eswar, K.K. Smart health monitoring system of patient through IoT. In Proceedings of the 2017 International Conference on I-SMAC (IoT in Social, Mobile, Analytics and Cloud) (I-SMAC); 2017; 551-556.

7. Kumar, R.; Rajasekaran, M.P. An IoT based patient monitoring system using raspberry Pi. In Proceedings of the 2016 International Conference on Computing Technologies and Intelligent Data Engineering (ICCTIDE'16); 2016; 1-4.

8. Ahouandjinou, A.S.R.M.; Assogba, K.; Motamed, C. Smart and pervasive ICU based-IoT for improving intensive health care. In Proceedings of the 2016 International Conference on Bio-engineering for Smart Technologies (BioSMART); 2016; 1-4.

9. Miller, J.C.; Skoll, D.; Saxon, L.A. Home Monitoring of Cardiac Devices in the Era of COVID-19. Curr. Cardiol. Rep. 2020, 23, 1, https://doi.org/10.1007/s11886-020-01431-w.

10. Wang, J.; Spicher, N.; Warnecke, J.M.; Haghi, M.; Schwartze, J.; Deserno, T.M. Unobtrusive Health Monitoring in Private Spaces: The Smart Home. Sensors 2021, 21.

11. Haghighatseir, N.; Ashrafi, H.; Rafiei, P.; Azadi, A. Dexamethasone ameliorates Alzheimer's pathological condition via inhibiting Nf-kB and mTOR signaling pathways. Biointerface Res. Appl. Chem. 2020, 10, 5556-5563.

12. Sahu, M.L.; Atulkar, M.; Ahirwal, M.K.; Ahamad, A. IoT-enabled cloud-based real-time remote ECG monitoring system. J. Med. Eng. Technol. 2021, 1-13, https://doi.org/10.1080/03091902.2021.1921870.

13. Kora, P.; Rajani, A.; Chinnaiah, M.C.; Swaraja, K.; Meenakshi, K. IoT Based Wearable Monitoring structure for detecting Abnormal Heart. In Proceedings of the 2021 International Conference on Sustainable Energy and Future Electric Transportation (SEFET); 2021; 1-4.

14. Lv, W.; Guo, J. Real-time ECG signal acquisition and monitoring for sports competition process oriented to the Internet of Things. Measurement 2021, https://doi.org/10.1016/j.measurement.2020.108359.

15. Ansari, S.; Farzaneh, N.; Duda, M.; Horan, K.; Andersson, H.B.; Goldberger, Z.D.; Nallamothu, B.K.; Najarian, K. A Review of Automated Methods for Detection of Myocardial Ischemia and Infarction Using Electrocardiogram and Electronic Health Records. IEEE Rev. Biomed. Eng. 2017, 10, 264-298, https://doi.org/10.1109/RBME.2017.2757953.

16. Sharma, L.D.; Sunkaria, R.K. Inferior myocardial infarction detection using stationary Wavelet transform and machine learning approach. Signal, Image Video Process. 2018, 12, 199-206, https://doi.org/10.1007/s11760-017-1146-z.

17. Meek, S.; Morris, F. ABC of clinical electrocardiography.Introduction. I-Leads, rate, rhythm, and cardiac axis. BMJ 2002, 324, 415-418, https://doi.org/10.1136/bmj.324.7334.415.

18. Bousseljot, R.; Kreiseler, D.; Schnabel, A. Nutzung der EKG-Signaldatenbank CARDIODAT der PTB über das Internet. 1995, 40, 317-318, https://doi.org/10.1515/bmte.1995.40.s1.317.

19. Goldberger, A.L.; Amaral, L.A.; Glass, L.; Hausdorff, J.M.; Ivanov, P.C.; Mark, R.G.; Mietus, J.E.; Moody, G.B.; Peng, C.K.; Stanley, H.E. PhysioBank, PhysioToolkit, and PhysioNet: components of a new research resource for complex physiologic signals. Circulation 2000, 101, E215-20, https://doi.org/10.1161/01.cir.101.23.e215.

20. Lu, H.L.; Ong, K.; Chia, P. An automated ECG classification system based on a neuro-fuzzy system. In Proceedings of the Computers in Cardiology 2000. 27 (Cat. 00CH37163); 2000; 387-390.

21. Thatipelli, T.; Kora, P. Classification of Myocardial Infarction using Discrete Wavelet Transform and Support Vector Machine. Int. Res. J. Eng. Technol. 2017, 4, 429-432.

22. Padhy, S.; Dandapat, S. Third-order tensor based analysis of multilead ECG for classification of myocardial infarction. Biomed. Signal Process. Control 2017, 31, 71-78, https://doi.org/10.1016/j.bspc.2016.07.007.

23. Sarkar, T.; Salauddin, M.; Hazra, S..; Choudhury, T.; Chakraborty, R. Comparative approach of artificial 
neural network and thin layer modelling for drying kinetics and optimization of rehydration ratio for bael (Aegle marmelos (L) correa) powder production. Econ. Comput. Econ. Cybern. Stud. Res. 2021, 55, 167184, http://dx.doi.org/10.24818/18423264/55.1.21.11.

24. Sarkar, T.; Salauddin, M.; Choudhury, T.; Um, J.S.; Pati, S.; Hazra, S.K.; Chakraborty, R. Spatial optimisation of mango leather production and colour estimation through conventional and novel digital image analysis technique. Spat. Inf. Res. 2021, 29, https://doi.org/10.1007/s41324-020-00377-z.

25. Arif, M.; Malagore, I.A.; Afsar, F.A. Automatic Detection and Localization of Myocardial Infarction Using Back Propagation Neural Networks. In Proceedings of the 2010 4th International Conference on Bioinformatics and Biomedical Engineering 2010; $1-4$.

26. Safdarian, N.; Dabanloo, N.; Attarodi, G. A New Pattern Recognition Method for Detection and Localization of Myocardial Infarction Using T-Wave Integral and Total Integral as Extracted Features from One Cycle of ECG Signal. J. Biomed. Sci. Eng. 2014, 7, 818-824.

27. Liu, W.; Huang, Q.; Chang, S.; Wang, H.; He, J. Multiple-feature-branch convolutional neural network for myocardial infarction diagnosis using electrocardiogram. Biomed. Signal Process. Control 2018, 45, 22-32, https://doi.org/10.1016/j.bspc.2018.05.013.

28. Han, C.; Shi, L. ML-ResNet: A novel network to detect and locate myocardial infarction using 12 leads ECG. Comput. Methods Programs Biomed. 2020, 185, 105138, https://doi.org/10.1016/j.cmpb.2019.105138.

29. Bhaskar, N.A. Performance Analysis of Support Vector Machine and Neural Networks in Detection of Myocardial Infarction. Procedia Comput. Sci. 2015, 46, 20-30, https://doi.org/10.1016/j.procs.2015.01.043.

30. Mukherjee, A.; Chatterjee, K.; Sarkar, T. Entropy-Aided Assessment of Amla ( Emblica officinalis ) Quality Using Principal Component Analysis. Biointerface Res. Appl. Chem. 2022, 12, 2162-2170, https://doi.org/10.33263/BRIAC122.21622170.

31. Jafarian, K.; Vahdat, V.; Salehi, S.; Mobin, M. Automating detection and localization of myocardial infarction using shallow and end-to-end deep neural networks. Appl. Soft Comput. 2020, 93, 106383, https://doi.org/10.1016/j.asoc.2020.106383.

32. Muminov, B.; Nasimov, R.; Mirzahalilov, S.; Sayfullaeva, N.; Gadoyboyeva, N. Localization and Classification of Myocardial Infarction Based on Artificial Neural Network. In Proceedings of the 2020 Information Communication Technologies Conference (ICTC) 2020; 245-249.

33. Liu, W.; Wang, F.; Huang, Q.; Chang, S.; Wang, H.; He, J. MFB-CBRNN: A Hybrid Network for MI Detection Using 12-Lead ECGs. IEEE J. Biomed. Heal. Informatics 2020, 24, 503-514, https://doi.org/10.1109/JBHI.2019.2910082.

34. Lui, H.W.; Chow, K.L. Multiclass classification of myocardial infarction with convolutional and recurrent neural networks for portable ECG devices. Informatics Med. Unlocked 2018, 13, 26-33, https://doi.org/10.1016/j.imu.2018.08.002.

35. Prabhakararao, E.; Dandapat, S. Myocardial Infarction Severity Stages Classification From ECG Signals Using Attentional Recurrent Neural Network. IEEE Sens. J. 2020, 20, 8711-8720, https://doi.org/10.1109/JSEN.2020.2984493.

36. Wang, H.M.; Zhao, W.; Jia, D.Y.; Hu, J.; Li, Z.Q.; Yan, C.; You, T.Y. Myocardial Infarction Detection Based on Multi-lead Ensemble Neural Network. Annu. Int. Conf. IEEE Eng. Med. Biol. Soc. IEEE Eng. Med. Biol. Soc. Annu. Int. Conf. 2019, 2019, 2614-2617, https://doi.org/10.1109/EMBC.2019.8856392.

37. Alghamdi, A.; Hammad, M.; Ugail, H.; Abdel-Raheem, A.; Muhammad, K.; Khalifa, H.S.; Abd El-Latif, A.A. Detection of myocardial infarction based on novel deep transfer learning methods for urban healthcare in smart cities. Multimed. Tools Appl. 2020, https://doi.org/10.1007/s11042-020-08769-x.

38. Gupta, A.; Huerta, E.; Zhao, Z.; Moussa, I. Deep Learning for Cardiologist-Level Myocardial Infarction Detection in Electrocardiograms. IFMBE Proc. 2021, 80, 341-355, doi:10.1007/978-3-030-64610-3_40.

39. Lih, O.S.; Jahmunah, V.; San, T.R.; Ciaccio, E.J.; Yamakawa, T.; Tanabe, M.; Kobayashi, M.; Faust, O.; Acharya, U.R. Comprehensive electrocardiographic diagnosis based on deep learning. Artif. Intell. Med. 2020, 103, 101789, https://doi.org/10.1016/j.artmed.2019.101789.

40. Darmawahyuni, A.; Nurmaini, S.; Sukemi Deep Learning with Long Short-Term Memory for Enhancement Myocardial Infarction Classification. In Proceedings of the 2019 6th International Conference on Instrumentation, Control, and Automation (ICA) 2019; pp. 19-23.

41. Tripathy, R.K.; Bhattacharyya, A.; Pachori, R.B. A Novel Approach for Detection of Myocardial Infarction From ECG Signals of Multiple Electrodes. IEEE Sens. J. 2019, 19, 4509-4517, https://doi.org/10.1109/JSEN.2019.2896308.

42. Fu, L.; Lu, B.; Nie, B.; Peng, Z.; Liu, H.; Pi, X. Hybrid Network with Attention Mechanism for Detection and Location of Myocardial Infarction Based on 12-Lead Electrocardiogram Signals. Sensors (Basel). 2020, 20, https://doi.org/10.3390/s20041020.

43. Gifari, M.W.; Zakaria, H.; Mengko, R. Design of ECG Homecare:12-lead ECG acquisition using single channel ECG device developed on AD8232 analog front end. In Proceedings of the 2015 International Conference on Electrical Engineering and Informatics (ICEEI) 2015; 371-376.

44. Analog Devices AD8232 Single-Lead ECG. Data Sheet 2013, 1-28.

45. Elgendi, M.; Fletcher, R.; Liang, Y.; Howard, N.; Lovell, N.H.; Abbott, D.; Lim, K.; Ward, R. The use of 
photoplethysmography for assessing hypertension. npj Digit. Med. 2019, 2, 60, https://doi.org/10.1038/s41746-019-0136-7.

46. Přibil, J.; Přibilová, A.; Frollo, I. First-Step PPG Signal Analysis for Evaluation of Stress Induced during Scanning in the Open-Air MRI Device. Sensors (Basel) 2020, 20, https://doi.org/10.3390/s20123532. 